\title{
Nature's origami
}

\author{
Understanding folding helps to analyze the self-structuring of molecules, organs and surfaces
}

\author{
Philip Hunter
}

A ll life from viruses to blue whales relies on folding processes that can have fatal consequences when they go wrong. At the molecular level, folding is involved in DNA packaging and the fundamental process by which proteins assume their structural conformation and thereby function. At the cellular and tissue level, many plants depend on folding to prevent pollen grains from drying up through evaporation, while for animals, it plays a crucial role in the development of various organs, including the gut and the mammalian brain. At all these levels, recent progress has yielded a better understanding of both the role and the process of folding, as well as how and why it can fail.

(c)

... brain folding evolved

because it was advantageous for neural processing of higher brain functions such as memory, perception, consciousness, language or thought."

The mammalian brain comprises an outer cerebral cortex, or gray matter, that is rooted on the sub-cortex, or white matter, which includes the underlying brainstem that connects to the spinal cord. Both the cerebral and sub-cortex are organized as sheets rather than a solid volume, and these sheets fold into convoluted shapes in many mammals. Ostensibly, brain folding evolved because it was advantageous for neural processing of higher brain functions such as memory, perception, consciousness, language, or thought. Selective pressures would work to increase the size of the neocortex where these functions take place, and, given its sheet-like structure, aim to maximize the surface area of the sheets. Selection would also need to optimize communication between neurons-just as with electronic computers, overall processing capability increases by reducing the distances signals have to travel—while facing physical limits to further increase brain size.

This is where folding comes into play, according to George Striedter, an evolutionary neurobiologist at the University of California, San Diego, USA, and Editor-in-Chief of Brain, Behavior and Evolution. "If we assume that it is adaptive to have the neocortex organized as a sheet, then there are only two ways to make it larger," he explained. "One is to have it form a fluid-filled balloon and the other is to fold it. Having a balloon would require having a very large head, which would be cumbersome both when it comes to giving birth and in adult animals, so folding is a better solution because it saves space. It also allows the connections between different parts of the cortex, and between the cortex and the brain to be relatively short. This saves volume and makes intra-brain communication faster."

ntriguingly, the development of the folded cortex is governed by physical mechanisms. In all mammals, the cerebral cortex initially develops as a smooth sheet driven by proliferation of neurons and outward radial migration along the surface [1]. Each of the cortex's two hemispheres at this stage is like the balloon Striedter described, kept inflated by intraventricular pressure from the production of cerebrospinal fluid. At the same time, the cortex remains rooted to the sub-cortex as it grows, which imposes constraints on its expansion, according to David Van Essen from the
Department of Anatomy \& Neurobiology at Washington University in St. Louis, USA, and one of the pioneers of brain folding. "I think a key issue is how many total cortical neurons there are relative to the total number of underlying subcortical neurons," he said. "If the cortical neurons vastly outnumber the subcortical neurons, taking average neuronal size into account as well, then the cortex will tend to fold as it wraps around the subcortical 'blob' [2].”

(c)

... folding itself is not an adaptation but merely a physical consequence that just emerged at a point in evolution."

Folding is therefore triggered by increasing tension along the axons that connect neurons on the cortical surface as it expands, and mediated by the fixation to the underlying neocortex. Local differences in folding can be accounted for by varying patterns of interconnectivity between neurons. The aggregate forces tend to bring strongly interconnected regions closer together, thereby forming a gyral fold in between, Van Essen explained, while weakly interconnected regions lack the tension to form a fold. Indeed, an earlier study had already established a relationship between the folding process during development and the relative growth of cortical and subcortical neurons [3]. "This very important and beautiful study accounts for much of what's going on in terms of stages of neurogenesis (with cortical neurons being born latest) and brain size (given that cortical proliferation lasts longer in bigger brains, producing more 
neurons and increasing the ratio of cortical to subcortical neurons)," Van Essen said. The bigger the ratio of cortical to subcortical neurons, the stronger the forces exerted on the surface as it wraps around the subcortex.

But despite growing understanding of the mechanisms that determine folding, the underlying physical rules have remained elusive. Now a Brazilian group, led by Bruno Mota and Suzana Herculano-Houzel from the Federal University of Rio de Janeiro, claimed they have found such a rule to describe the cortical folding process in all mammals [4]. Their study started with the observation that although cortexes are three-dimensional, their folding can be regarded as a two-dimensional fractal process that, analogous to the crumpling of paper, generates repeating patterns at eversmaller dimensions which are determined by the sheet's surface area and thickness. The Brazilian researchers proposed that the same physical rule governs folding in brains, which is defined by the surface area of the cortex divided by the square root of its thickness.

A key measure is the degree of folding: The ratio between the surface and the area that a balloon shape of the same total volume would occupy. The greater the amount of folding, the larger the ratio, which in turn is an indicator of the brain's processing capability for a given volume. Yet, while the degree of folding tends to scale both with the volume of the brain and the number of neurons it contains, the relationship is not exact. The cortical folding index in elephants is twice that of humans despite the fact that the elephant cortex only contains a third as many neurons. Size also fails to predict folding at all: The brain of the baboon is highly folded while that of the manatee, which is the same size, is almost entirely smooth.

Mota and Herculano-Houzel therefore analyzed data about mammalian brains across orders and clades and found that, while all brains below a certain volume or with fewer than 30 million cortical neurons do not fold, the relationship varied between animal orders. The folding index also varies for a given number of neurons: It is for instance larger among artiodactyls (eventoed ungulates such as giraffes, sheep, and pigs) than among primate species. Eventually, the authors came up with their formula associating folding index solely with cortical surface area and thickness. If a sheet of paper were infinitely thin, it would go on folding indefinitely in a fractal pattern. In practice though, the thickness of the paper prevents further folding beyond a certain point and exactly the same holds true for the mammalian brain, the authors claim.

The question then is how evolution and selective factors have driven brain folding to increase the surface area within a limited volume. Mota, in common with other specialists in the field, believes that folding itself is not an adaptation but merely a physical consequence that just emerged at a point in evolution. "We do not think folding was a specific adaptation that evolved," he said. But having emerged, there was scope for manipulating it by changing the thickness of the cortex and surface area. "Evolution is free to fiddle with the total area, the thickness and the number of neurons and, presumably, the specific evolutionary pressures under which each species evolved will lead to different values of each being selected," Mota explained. "So some mammals have very thick cortices, with the manatee being an extreme case and, as a result, having a smooth cortex, while others have thinner ones."

Yet, not everyone is ready to accept Mota and Herculano-Houzel's theory. Van Essen is unconvinced by the suggested mechanism on the grounds that it fails to account for structural differences within species and between regions of individual brains. He argued that in the cerebral cortex, tension along axons in the white matter explains how and why the cortex folds in a pattern that is specific to species. Since then evidence has accumulated to support this view, van Essen believes. "Thickness variations within a species, which were not even discussed by Mota and Herculano-Houzel, can in principle be explained by regional differences in neuronal architecture," he said. "Cortical regions that are thick tend to have neurons with larger dendrite arbors, which are more isotropic and I propose less dominated by radially elongated processes."

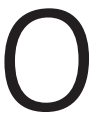
n a similar physical scale to the brain, the gut in vertebrates also folds as it develops within the growing organism. As the gut tube grows into the body cavity, it forms a reproducible looped pattern, but the mechanisms governing this pattern formation were unclear until a recent paper demonstrated that the process results from differential growth between the gut and the underlying dorsal mesenteric sheet tissue [5]. "We were able to build a rubber mimic of this process and provide some simple formulas to predict the size and number of the loops from mechanical properties of the tissue," said study author Thierry Savin, University Lecturer in Bioengineering at Cambridge University, UK (Fig 1). The team also developed a mathematical theory and computational model to predict the number, size, and shape of intestinal loops based solely on the measurable geometry, elasticity, and relative growth of the tissues. This model successfully predicted gut folding in
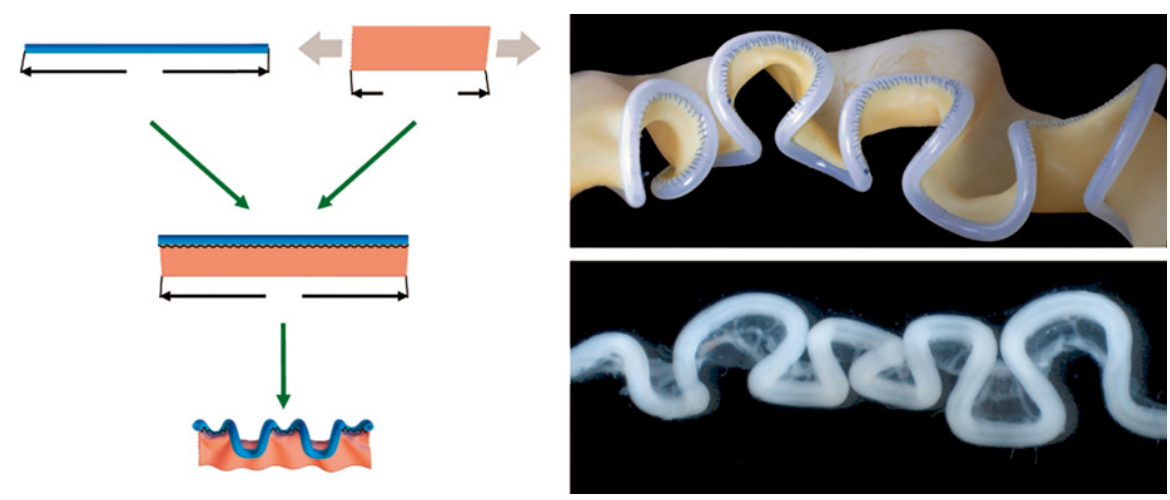

Figure 1. Simulation of gut morphogenesis.

A thin rubber sheet was stretched uniformly along its length and then stitched to a unstretched rubber tube; the differential strain mimics the differential growth of the two tissues (left). On relaxation, the composite rubber model deformed into a structure very similar to the chick gut (upper right). Lower right: chick gut at embryonic day E12. The superior mesenteric artery has been cut out. Adapted from [5] with permission from Nature. 
several diverse species, including quails and mice. The basic principles here are very similar to brain folding.

\section{"Convergent evolution is probably also manifest in folding of pollen grains, which have a similar fundamental structure across diverse taxa"}

"The main advance in my opinion was to isolate the physical context of folding and describe it with very few parameters," said Savin. "From a biological point of view, embryonic development is an extremely complicated process, likely to involve a large amount of genes and signaling. It is thus very valuable to reduce this problem to a few important parameters, and focus on the fewer genes tuning these material properties." Such genes might have been subject to selective forces, aiming to optimize the gut folding process by tuning the relative rates of growth of the tube and dorsal sheet.

$\mathrm{D}$ ifferential growth and folding also crops up in the plant kingdom, for example, during the flowering process of the lily, which creates curvature of the petals. A 2011 paper overturned the previous hypothesis that this process was controlled by the relative growth of the inner layers of petals along the structural rib down their middle [6] and presented evidence that, instead, petal curvature was governed by differential growth between the petals' edges, which wrinkle as the flower opens. This would bring the theory of flower opening into line with leaf development, suggesting that both processes are subject to convergent evolution, as the paper's authors argue.

Convergent evolution is probably also manifest in folding of pollen grains, which have a similar fundamental structure across diverse taxa. Pollen grains have a tough impermeable outer shell, the exine that is punctuated with soft spots, or apertures, through which germination occurs. The apertures also allow moisture to enter and leave, causing swelling or shrinking of the grain. This has long been known, but it was unclear how the pollen grains avoided shriveling up completely under dry conditions. The answer lies in folding as a Chilean study found out [7].
"The main mathematical principle behind the folding of pollen grains is the use of these strategically placed 'soft spots' or apertures to favor a mode of inextensional deformation that would not be observed otherwise," explained Jacques Dumais, from the Universidad Adolfo Ibáñez in Chile and one of the authors. "Without these carefully designed apertures, the pollen grain would continually lose water and crumple like a raisin without ever being able to seal itself. With the apertures in place, the pollen grain folds neatly thus covering all the surfaces that are liable to let water diffuse out of the grain." The necessity for such folding has presumably exerted a constraint on pollen evolution as the process appears to be conserved across many taxa.

F olding also plays a crucial role at the molecular level to organize the threedimensional structure of DNA and proteins. The dynamics at these scales are very different as the predominant forces are electrostatic attraction and van der Waals forces, but there are some common underlying principles such as the tendency to form folds that are as stable as possible and keep different forces in equilibrium. Recent work again unraveled how evolution operates on two key aspects of protein folding - the folding process itself and the purpose of the protein - to describe the role of direct versus indirect selection on protein structure, function, and its ability to interact with other molecules. Direct selection operates on an existing trait that confers an intrinsic advantage for a cell, while indirect selection creates a new trait by chance that subsequently may be recruited if it turns out to be useful. Such traits evolving in the absence of direct selective pressure are known as spandrels and one question in biology is the role of these almost accidental developments. A paper published this year suggested that, in the case of protein folding, spandrels have been more important than previously thought [8]

"The reason folding and binding can evolve as spandrels is because they are not independent properties of a protein: it can only bind when folded," explained Michael Manhart, one of the study's two authors at Harvard University in the USA. "Thus, a protein can effectively make itself fold more stably if it binds strongly to something, because that will keep it in its folded state, a phenomenon we refer to as 'bindingmediated stability’. Conversely and more obviously, it binds more strongly if it folds stably. Thus, the structural coupling of these traits is key to them evolving as spandrels."

Their paper then illustrates how evolution can produce stably folding proteins even when there is direct selection for binding only, and conversely, how evolution can produce strong binding proteins even when there is direct selection for folding only. "There are various selective mechanisms that could produce direct selection only for one trait or the other," said Manhart. "The first case is straightforward: there is direct selection for binding only as long as misfolded proteins are not inherently harmful to the cell. However, it is wellestablished that at least some proteins are indeed toxic to cells when misfolded due to their propensity to aggregate."

\section{"One common theme of these advances in understanding the evolution, role and mechanisms of folding at different scales is the obvious applicability"}

Another paper directly illustrated this misfolding toxicity by expressing nonfunctional proteins in yeast and observing a significant fitness cost if those proteins acquired destabilizing mutations [9]. "Thus, direct selection for folding should occur for proteins that are toxic when misfolded, and thus they could possibly evolve strong but nonfunctional binding interactions solely to prevent them from misfolding," Manhart added. Such binding interactions could become spandrels that might come in useful later.

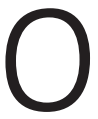
ne common theme of these advances in understanding the evolution, role, and mechanisms of folding at different scales is the obvious applicability, for example, for therapeutics or biomimetic materials. A number of major diseases, such as Alzheimer's or Creutzfeld Jacob, result from protein misfolding in the brain, while Lissencephaly, or literally smooth brain, is caused by the failure of cortical folding through defective neuronal migration during early brain development. Unraveling the mechanisms behind these might help in developing therapeutic interventions, 
whereas folding mechanisms in plants could inspire biomimetics, such as unidirectional valves, by exploiting the mechanism of folding in pollen grains to shut down water loss. Beyond these practical possibilities, folding is an intriguing example of how evolution adopted basic physical mechanisms on various scales.

\section{References}

1. Desmond ME, Jacobson AG (1977) Embryonic brain enlargement requires cerebrospinal fluid pressure. Deu Biol 57: 188-198

2. Van Essen DC (1997) A tension-based theory of morphogenesis b0300 and compact wiring in the central nervous system. Nature 385: $313-318$

3. Finlay BL, Darlington RB (1995) Linked regularities in the development and evolution of mammalian brains. Science 268: 1578-1584

4. Mota B, Herculano-Houzel S (2015) Cortical folding scales universally with surface area and thickness, not number of neurons. Science 349: $74-77$

5. Savin T, Kurpios NA, Shyer AE, Florescu P, Liang $H$, Mahadevan L, Tabin CJ (2011) On the growth and form of the gut. Nature 476: 57-62

6. Liang H, Mahadevan L (2011) Growth, geometry and mechanics of the blooming lily. Proc Natl Acad Sci USA 108: 5516-5521
7. Katifori E, Alben S, Cerda E, Nelson DR, Dumais J (2010) Foldable structures and the natural design of pollen grains. Proc Natl Acad Sci USA 107: $7635-7639$

8. Manhart M, Morozov AV (2015) Protein folding and binding can emerge as evolutionary spandrels through structural coupling. Proc Natl Acad Sci USA 112: 1797-1802

9. Geiler-Samerotte KA, Dion MF, Budnik BA, Wang SM, Hartl DL, Drummond DA (2011) Misfolded proteins impose a dosagedependent fitness cost and trigger a cytosolic unfolded protein response in yeast. Proc Natl Acad Sci USA 108 $680-685$ 